American Journal of Pharmaceutical Education 2018; 82 (10) Article 7009.

\title{
COMMENTARY
}

\section{Identifying Core Content for Electrocardiogram Instruction in Doctor of Pharmacy Curricula}

\author{
Zachary R. Noel, PharmD, , Craig J. Beavers, PharmD, ${ }^{\mathrm{b}}$ Steven P. Dunn, PharmD, ${ }^{c}$ \\ Anne Marie Schullo-Feulner, PharmD, ${ }^{\mathrm{d}}$ Lauren Caldas, PharmD, ${ }^{\mathrm{e}}$ Dave L. Dixon, PharmD \\ ${ }^{a}$ University of Maryland School of Pharmacy, Baltimore, Maryland \\ ${ }^{\mathrm{b}}$ University of Kentucky Healthcare, Lexington, Kentucky \\ ${ }^{\mathrm{c}}$ University of Virginia Health System, Charlottesville, Virginia \\ ${ }^{\mathrm{d}}$ University of Minnesota College of Pharmacy, Minneapolis, Minnesota \\ e Virginia Commonwealth University School of Pharmacy, Richmond, Virginia \\ Submitted February 19, 2018; accepted March 31, 2018; published December 2018.
}

\begin{abstract}
Minimum competencies for diagnostic tools, such as the electrocardiogram, are not well-defined in current standards or publications. The electrocardiogram has significant pharmacotherapeutic implications that pharmacists should have an adequate understanding of. This commentary highlights the importance of pharmacists' understanding of key elements of the electrocardiogram and drafts a set of recommended minimum competencies for graduating pharmacy students.
\end{abstract}

Keywords: electrocardiogram, cardiology, arrhythmias

Diagnostic test results often drive pharmacotherapeutic decision making and are integral to measuring efficacy or toxicity of medications. As a rudimentary example, understanding and interpreting blood pressure readings is fundamental to managing pharmacotherapy for the treatment of hypertension. The objective data obtained from a blood pressure reading directly affects whether treatment is started, titrated, or discontinued. Although more complex and less objective than a blood pressure reading, the electrocardiogram (ECG) is a cornerstone diagnostic tool within cardiology and is used to determine pharmacologic and non-pharmacologic treatment of cardiac arrhythmias, and to monitor for safety and toxicity of both cardiovascular and non-cardiovascular medications. Despite the essential information provided by ECGs and many other diagnostic tools, the Accreditation Council for Pharmacy Education Standards do not provide sufficient guidance on whether such tools should be incorporated into the Doctor of Pharmacy (PharmD) curriculum. ${ }^{1}$

Similarly, while previous publications have highlighted novel instructional methods for teaching ECGs to pharmacy students, none of them addresses what the minimum competency should be for graduating pharmacy students. ${ }^{2,3}$ Given the lack of existing data and guidance on this topic, this commentary will focus on the importance

Corresponding Author: Zachary R. Noel, University of

Maryland School of Pharmacy, 20 N. Pine St., Baltimore, MD

21201. Tel: 410-706-5819. E-mail: znoel@rx.umaryland.edu and relevance of teaching basic understanding and interpretation of ECGs and will recommend minimum competencies for graduating pharmacy students.

\section{The Relevance of Understanding and Interpret- ing an ECG}

The ECG is one of the most critical and rapidly used non-invasive tests in cardiology care today. An ECG is a report of electrical conduction in the heart that is obtained through placing multiple leads on the chest and extremities. Although a host of information about the heart can be garnered from an ECG, Table 1 lists basic findings from an ECG report that are foundational in understanding conduction abnormalities. For students, understanding lead placement, the difference between a basic rhythm strip and a 12-lead ECG, and the basic science behind ECGs (eg, a record of timing and strength of the heart's electrical signals through voltage-gated sodium, potassium and calcium channels) is critical to comprehending and contextualizing the findings. Having this basic understanding in place allows clinicians to use this information to interpret basic ECG findings and apply them to patients.

For pharmacists, understanding these basic concepts is important in different settings and environments. Tables 2 and 3 provide a list of common drug-induced ECG changes and changes that require rapid identification and interpretation by a health care professional, respectively. The most obvious example of when pharmacists may use 
American Journal of Pharmaceutical Education 2018; 82 (10) Article 7009.

Table 1. Key ECG Information

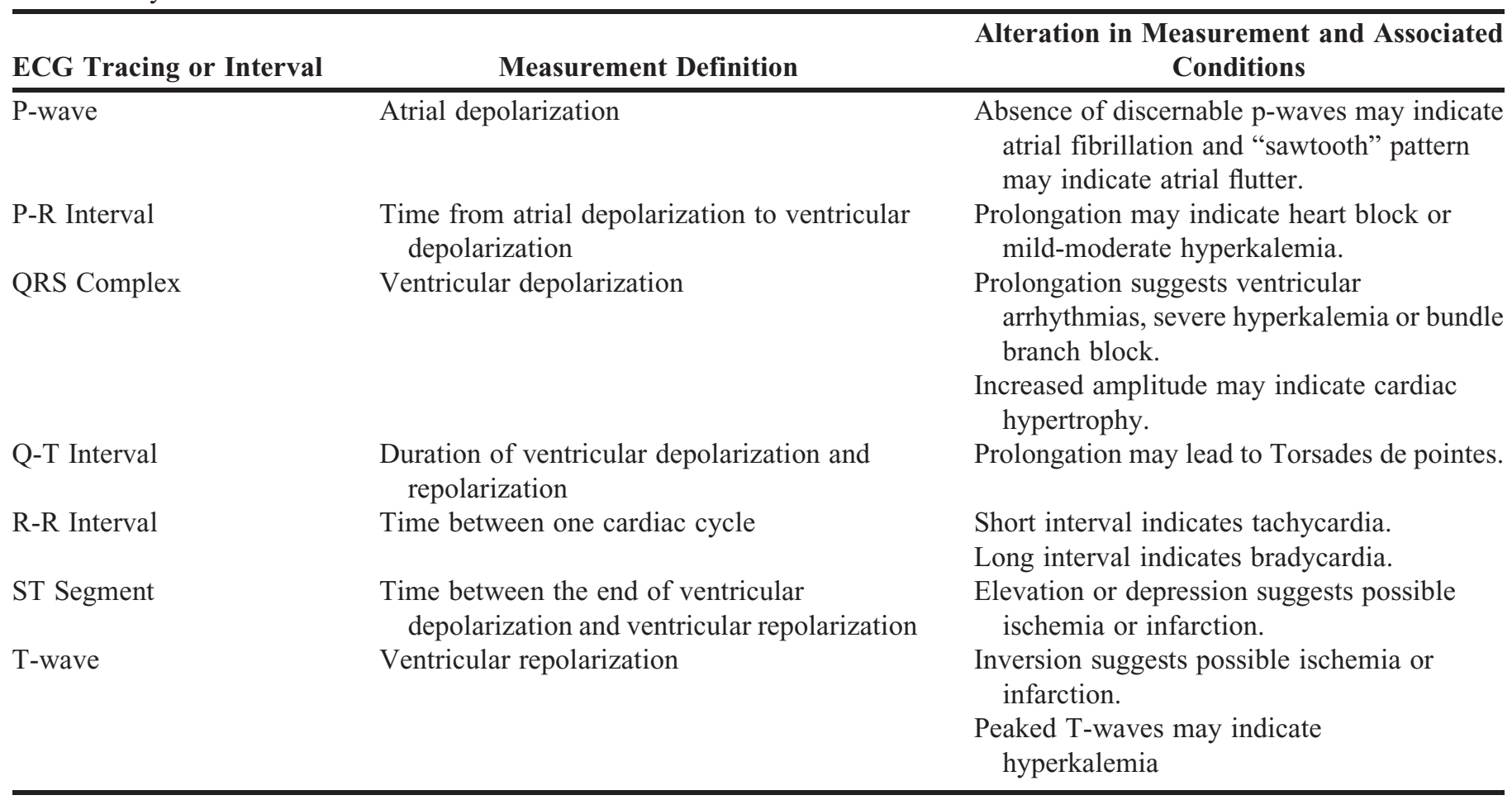

these skills is in the emergency setting. Many pharmacists working in acute care environments, and in particular those who work in emergency departments and intensive care units, are routinely faced with emergency scenarios. The pharmacist's role in cardiac emergencies is recognized as part of the chain of survival in the 2015 Advanced Cardiac Life Support guidelines. ${ }^{4}$ Pharmacist participation during advanced cardiac life support can promote safe drug preparation and prompt administration, and in some instances, may alert the team to cardiac rhythm changes. The Institute for Safe Medication Practices has encouraged pharmacist participation in the management of cardiac arrests to reduce the risk of medication-related errors. ${ }^{5}$ In fact, medication errors during code responses are nearly 40 times more likely to result in harm and 51 times more likely to result in death compared to non-emergent-related medication errors. ${ }^{6}$ In addition, the Heart Rhythm Society's 2015 Statement on Clinical Cardiac Electrophysiology specifically mentions pharmacists as a required health care practitioner for developing and maintaining adequate electrophysiology training. ${ }^{7}$

While not all graduating pharmacy students will be practicing in the acute care setting, most will be in work environments that require an understanding of drug-induced ECG changes. As a common example, consider community pharmacists alerted for concomitant QT prolonging medications. While QT prolongation and the

Table 2. Selected Examples of Drug-Induced ECG Changes and Arrhythmias

\section{ECG Findings}

PR Prolongation

QT/QTc Prolongation

QRS Prolongation

Ventricular Arrhythmias

(monomorphic or polymorphic)

Supraventricular Tachycardia

\section{Examples of Inciting Drugs}

Beta-blockers, digoxin, non-dihydropyridine calcium channel blockers

Antipsychotics (eg, haloperidol, ziprasidone), fluorquinolones, antiarrhythmics (eg, sotalol, dofetilide), antiemetics (eg, ondansetron), methadone, others

Antiarrhythmics (eg, procainamide, flecainide), tricyclic antidepressants (eg, amitriptylline)

Digoxin, stimulants, catecholamines, QT prolonging medications, antiarrhythmics (eg, flecainide)

Caffeine, stimulants 


\section{American Journal of Pharmaceutical Education 2018; 82 (10) Article 7009.}

Table 3. Key ECG Changes Requiring Rapid Identification

Monomorphic Ventricular Tachycardia

Polymorphic Ventricular Tachycardia

Ventricular Fibrillation

Pulseless Electrical Activity

ST-Elevation Myocardial Infarction

Symptomatic bradycardia or complete heart block

Atrial fibrillation causing hemodynamic instability

subsequent risk of Torsades de pointes is of grave consequence, pharmacists should be able to differentiate whether these warnings warrant action or not. ${ }^{8}$ A pharmacist lacking the basic understanding of drug-induced QT prolongation and risk factors for Torsades de pointes will either burden local providers with unnecessary calls, or worse, ignore a warning that could result in patient harm. ${ }^{9}$ There are also examples of the community pharmacist's evolving role in early recognition of arrhythmias. In Canada, patients can ask for an ECG screening in community pharmacies. One study found screening for atrial fibrillation in community pharmacies to be a cost-effective strategy to reduce the burden of stroke. ${ }^{10}$ Other reports have shown routine screening for atrial fibrillation by free pulse-checks to be an effective way to identify patients with asymptomatic atrial fibrillation. ${ }^{11}$ Pharmacist referral for ECG and anticoagulation therapy in this setting could have tremendous impact on patient outcomes.

What minimum competency should students be expected to have upon graduation? Although routine ECG interpretation is uncommon for many health care professionals, having an understanding and ability to recognize and interpret pertinent ECG changes remains a point of emphasis in numerous health care education programs. For example, nursing schools emphasize early recognition of cardiac arrest and life-threatening cardiac arrhythmias, even though many nurses pursue career paths that do not require routine ECG interpretation. ${ }^{12}$ Similarly, all pharmacists do not work in an environment that routinely requires them to interpret ECG changes; however, baseline knowledge and understanding of ECGs is important for the many pharmacy students entering residency or positions that provide direct patient care. For instance, pharmacists in ambulatory settings should be able to recognize when an ECG is indicated when the clinical symptoms (eg, chest pain, syncope, palpitations) are suggestive of an arrhythmia.

The diverse professional opportunities and career paths available for graduating pharmacy students make defining minimum competencies a difficult task. Recently, the American Association of Colleges of Pharmacy (AACP) drafted a list of Entrustable Professional
Table 4. Minimum Competencies for ECG Understanding and Interpretation for Graduating Pharmacy Students

Explain the electrical properties of human cells and tissue (eg, ion channels, action potentials).

Describe the electrical conduction system of the heart and how it correlates to an ECG tracing (eg, atrial depolarization is the p-wave on an ECG).

Discuss the process of performing ECG monitoring (eg, lead placement, electrocardiograph).

Recognize the most common conduction abnormalities observed in clinical practice and potentially life-threatening arrhythmias (eg, atrial fibrillation, ventricular fibrillation, etc.).

Assess symptoms that would warrant an indication or referral for an ECG.

Identify and resolve clinically significant drug-induced ECG changes.

Activities (EPAs) that graduating pharmacy students might be entrusted to perform independently. ${ }^{13}$ The relevant EPA for understanding or interpreting an ECG is the "Patient Care Provider Domain: Analyze information to determine the effects of medication therapy, identify medication-related problems, and prioritize healthrelated needs." Examples included within this EPA are measuring vital signs and blood pressure. Although there is no mention of ECGs within this EPA, it should be noted that listed examples of supporting tasks are provided by AACP as suggestions and are not meant to be all-inclusive. It should also be noted that EPAs are set forth to be a baseline, not a ceiling, for trainees. As such, many pharmacy schools train their students beyond listed EPAs.

The most likely application of understanding ECGs in context of the aforementioned EPA is as it relates to medication-related problems. As listed in Table 2, druginduced ECG changes are pervasive among cardiovascular and non-cardiovascular medications. Appreciating the significance of drug-induced ECG changes and their potential implications is vital to ensuring safe medication use, regardless of which sector of pharmacy students enter after graduation. As such, minimum expectations should be set forth for graduating pharmacy students. The drafted recommendations may serve as a list of minimum competencies for pharmacy schools to use (Table 4). Some schools may choose to require more than these minimum competencies, and schools placing an emphasis on residency preparation may desire to incorporate additional ECG training into the curriculum. Likewise, student pharmacists with an interest in cardiology, critical care, or emergency medicine who are considering specialty residency training may pursue opportunities to hone their understanding and interpretation of ECGs through elective 


\section{American Journal of Pharmaceutical Education 2018; 82 (10) Article 7009.}

experiences or select advanced pharmacy practice experiences, if available.

As pharmacy practice continues to evolve, so will minimum competencies for graduating pharmacists. Although pharmacists have a limited role in diagnosing disease, there is a responsibility for pharmacists to understand common diagnostic tests, which greatly influence pharmacotherapy decisions. The extent to which pharmacists should be responsible for diagnostic tests such as the electrocardiogram is not well characterized in current standards. We advocate that pharmacy schools establish minimum competencies for understanding and interpreting ECGs to ensure pharmacy students graduate with a fundamental understanding of their role in monitoring drug therapy. Additional work is needed to better understand best practices for ensuring these competencies are met.

\section{REFERENCES}

1. Accreditation Council for Pharmacy Education. Accreditation standards and key elements for the professional program in pharmacy leading to the doctor of pharmacy degree. Standards 2016. https://

www.acpe-accredit.org/pdf/Standards2016FINAL.pdf.

2. Bose DD. An elective course in cardiovascular electrophysiology for pharmacy learners. Am J Pharm Educ. 2016;80(8):Article 130.

3. Schultz K, Brackbill M. Teaching electrocardiogram basics using dance and movement. Am J Pharm Educ. 2009;73(4):

Article 70 .

4. Kronick S, Kurz M, Welsford M, et al. Part 4: systems of care and continuous quality improvement: 2015 American Heart Association guidelines update for cardiopulmonary resuscitation and emergency cardiovascular care. Circulation. 2015;132(18):S397-S413.

5. Institute for Safe Medication Practices. Preventing medication errors during codes. https://www.ismp.org/resources/preventingmedication-errors-during-codes. Accessed January 16, 2018. 6. Lipshutz A, Morloc L, Winters B, et al. Medication errors associated with code situations in U.S. hospitals: direct and collateral damage. Jt Comm J Qual Patient Saf. 2008;34(1):46.

7. Zipes DP, Calkins H, Daubert JP, et al. 2015 ACC/AHA/HRS advanced training statement on clinical cardiac electrophysiology (a revision of the ACC/AHA 2006 update of the clinical competence statement on invasive electrophysiology studies, catheter ablation, and cardioversion). Heart Rhythm. 2016;13(1):e3-e37.

8. Indermitte J, Beutler M, Bruppacher R, Meier CR, Hersberger KE. Management of drug-interaction alerts in community pharmacies. $J$ Clin Pharm Ther. 2007;32(2):133-142.

9. van der Sijs H, Kowlesar R, Klootwijk A, Nelwan S, Vulto A, van Gelder T. Clinically relevant QTc prolongation due to overridden drug-drug interaction alerts: a retrospective cohort study. $\mathrm{Br}$ J Clin Pharmacol. 2009;67(3):347-354.

10. Tarride JE, Dolovich L, Blackhouse G. Screening for atrial fibrillation in Canadian pharmacies: an economic evaluation. CMAJ Open. 2017;5(3):E653-E661.

11. Antoniou S, Papastergiou J, de Rango F, et al. Benefits of active involvement of community pharmacists in know your pulse awareness campaign. Euro Heart J. 2017;38(Suppl 1).

12. Varvaroussis DP, Kalafati M, Pliatsika P, Castrén M, Lott C, Xanthos T. Comparison of two teaching methods for cardiac arrhythmia interpretation among nursing students. Resuscitation. 2014;85(2):260-265.

13. Haines S, Pittenger A, Stolte SK, et al. Core entrustable professional activities for new pharmacy graduates. Am J Pharm Educ. 2017;81(1):Article S2. 